\section{Maturation of neurotransmission in the developing rat cochlea: immunohistochemical evidence from differential expression of synaptophysin and synaptobrevin 2}

\author{
S. He, J. Yang \\ Department of Otorhinolaryngology- \\ Head \& Neck Surgery, Xinhua Hospital, \\ Shanghai Jiaotong University, Shanghai \\ Jiaotong University Ear Institute, China
}

\section{Abstract}

Synaptophysin and synaptobrevin 2 associate closely with packaging and storage of synaptic vesicles and transmitter release, and both play important roles in the development of rat cochlea. We examined the differential expression of synaptophysin and synaptobrevin 2 in the developing Sprague-Dawley rat cochlea, and investigated the relationship between their expression and auditory development. The expression of synaptophysin and synaptobrevin 2 was not observed in Kolliker's and Corti's organ at postnatal 1 day (P1) and $\mathrm{P} 5$, and the top turn of the cochlea at P10. Expression was detected in the outer spiral bundle (OSB), the inner spiral bundle (ISB), and the medial wall of the Deiters' cell of the cochlea at P14, and P28, and in the middle or the basal turn of Corti's organ at P10. Synaptobrevin 2 was expressed in the top of the inner hair cells (IHCs) in Corti's organ of both P14 and P28 rats. All spiral ganglion neurons (SGNs) were stained at all ages examined. The localization of synaptophysin and synaptobrevin 2 in the cochlea was closely associated with the distribution of nerve fibers and neural activity (the docking and release of synaptic vesicles). Synaptophysin and synaptobrevin 2 were expressed in a dynamic manner during the development of rat cochlea. Their expression differences during the development were in favor of the configuration course constructed between nerve endings and target cells. It also played a key role in the formation of the correct coding of auditory information during auditory system development.

\section{Introduction}

The development of the auditory system is a tightly regulated complex process. in which various kinds of structural proteins such as connexin, myosin and parvalbumin, are expressed in specific areas of cochlea and play important roles. The development of the rat auditory system lasts from the embryonic period to 14 days after birth. ${ }^{1,2}$. The maturation of the auditory system has been shown to be affected by the external environment, hormones and other factors after birth.,4

Synaptic vesicles are multi-protein complexes composed of a number of conserved proteins. Synaptophysin and synaptobrevin 2 are both membrane bound proteins found in synaptic vesicles and are important for packaging, storage, and release of neurotransmitters. ${ }^{5,6}$ Synaptophysin is a synaptic vesicleassociated protein that provides specific labeling of olivocochlear efferent fibers and terminals in the cochlea. ${ }^{7}$ Synaptobrevin 2 is one of the major components of the soluble N-ethylmaleimide-sensitive factor attachment protein receptor (SNARE) complex, and plays a key role in vesicle docking and fusion. ${ }^{8}$ To date, the only non-SNARE binding partner of synaptobrevin 2 identified is synaptophysin. ${ }^{9}$

Synaptophysin is a major resident of the synaptic vesicle membrane, and its binding to synaptobrevin 2 is specific and exclusive. ${ }^{10}$ As a regulatory protein, synaptophysin can regulate the utilization of synaptobrevin 2 in the formation of the SNARE complex before vesicles fuse with the cell membrane. ${ }^{11}$ Thus, this interaction plays an important role in the metabolism of synaptic vesicles. It was previously found that synaptophysin and synaptobrevin 2 are expressed extensively in the cochlea in areas such as the spiral bundle, ${ }^{12-14}$ peripheral support cells and spiral ganglion neurons (SGNs). ${ }^{8}$ Using RT-PCR and in situ hybridization, synaptophysin and synaptobrevin 2 were confirmed to be expressed in the cochleae of rodent. ${ }^{15-17}$ However, these results were found in mature cochleae; the expression patterns and mechanism of interaction in the developing rat cochlea are still not clear.

In the present study, we have attempted to characterize the expression of synaptophysin and synaptobrevin 2 in the developing Sprague-Dawley rat, in hopes of understanding the potential significance of synaptophysin and synaptobrevin 2 interactions in cochlear maturation.

\section{Material and Methods}

\section{Specimen preparation}

Sprague-Dawley rats (Super-B\&K, China), which were one day (P1), five days (P5), ten days (P10), 14 days (P14) and 28 days (P28) after parturition, were chosen (5 rats in each age group). All adult rats or the paternal parents of infant rats had a normal pinna reflex.
Correspondence: J. Yang, Department of Otorhinolaryngology-Head \& Neck Surgery, Xinhua Hospital, Shanghai Jiaotong University Ear Institute, Shanghai 200092, China.

Tel. +86.21.25078532 - Fax: +86.21.65156489

E-mail: yangjun_bioon@126.com

Key words: neurotransmission; synaptophysin; synaptobrevin 2; cochlear development; synapse

Contributions: SH carried out the laboratory experiments, analyzed and interpreted the results, discussed analyses and wrote the paper; JY defined the research theme and designed methods. All authors approved the version to be published.

Conflict of interest: the authors declare no potential conflict of interest.

Acknowledgements: this investigation was supported by the National Natural Science Foundation of China (no. 30772396).

Received for publication: 1 July 2010 . Accepted for publication: 12 November 2010.

This work is licensed under a Creative Commons Attribution 3.0 License (by-nc 3.0).

CC Copyright S. He and J. Yang, 2011

Licensee PAGEPress, Italy

European Journal of Histochemistry 2011; 55:e2 doi:10.4081/ejh.2011.e2

All experiments conformed to the regulations of Shanghai Animal Management Committee. The rats were decapitated and the cochleae were harvested from the temporal bones and further microdissected in cold $4 \%$ paraformaldehyde. The cochleae were then perfused through the round window with the same fixative solution, post-fixed overnight and then rinsed for 24-240 $\mathrm{h}$ in phosphate buffer containing 10\% EDTA and $4 \%$ paraformaldehyde. They were then washed for $3 \mathrm{~h}$ in phosphate buffer containing $15 \%$ sucrose followed by phosphate buffer containing $30 \%$ sucrose overnight. The cochleae were frozen in optimum cutting temperature compound (Sakura, Torrance, CA, USA) at $4^{\circ} \mathrm{C}$ and cut on a cryostat into $10 \mu \mathrm{m}$-thick sections along the modiolus. The glass slides were precoated with poly-D-lysine to avoid detachment. The sections were stored at $-20^{\circ} \mathrm{C}$ until use.

\section{Immunohistochemistry}

Frozen sections were dried for $2 \mathrm{~h}$ at room temperature. After three rinses with phosphatebuffered saline (PBS, pH 7.4), the sections were incubated in PBS supplemented with $0.1 \%$ Triton X-100 for $40 \mathrm{~min}$ at $37^{\circ} \mathrm{C}$ and then with a blocking solution containing $10 \%$ normal goat serum (Sigma-Aldrich, St. Louis, MO, USA) for 
30 min at room temperature. Incubation with a mixture of monoclonal and polyclonal antibodies against synaptophysin (1:200, Mouse Monoclonal Anti-Synaptophysin Receptor; Sigma-Aldrich, St. Louis, MO, USA) and synaptobrevin 2 (1:200, Rabbit Polyclonal Anti- synaptobrevin 2 Receptor; Abcam, Cambridge, UK) diluted in PBS was performed overnight at $4^{\circ} \mathrm{C}$.
For the control group, PBS was used instead of primary antibodies. Immunofluorescence was detected with Alexa Fluor $555 \quad \mathrm{~F}\left(\mathrm{ab}^{\prime}\right) 2$ Fragment-labeled goat anti-mouse antibody (1:400, Invitrogen, Carlsbad, CA, USA) and FITC-labeled goat anti-rabbit antibody (1:100, Beyotime, Haime, China) and analyzed by fluorescence microscopy (Leica, Wetzlar, Germany).
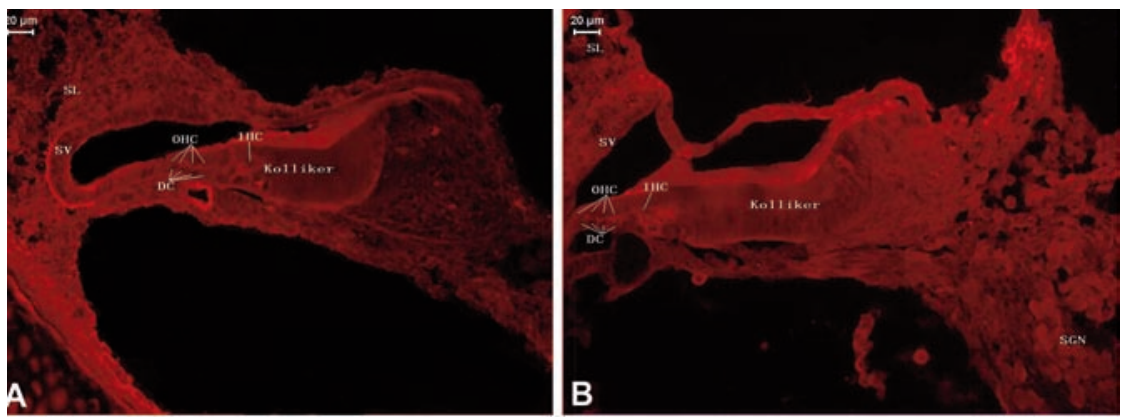

Figure 1. Immunostaining of synaptophysin in Corti's organ and Kolliker's organ of P1 and P5 group. (A) 200x. There are immature supporting cells and hair cells in Corti's organ and Kolliker's organ of cochleae. Positive staining of synaptophysin is not found in Corti's organ and Kolliker's organ in P1 group. (B) 200x. Positive staining of synaptophysin is not found in Corti's organ and Kolliker's organ in P5 group either. (IHC, inner hair cell; OHC, outer hair cell; DC, deiters cell; SV, stria vascularis; SL, spiral ligament; SGN: spiral ganglia neuron).
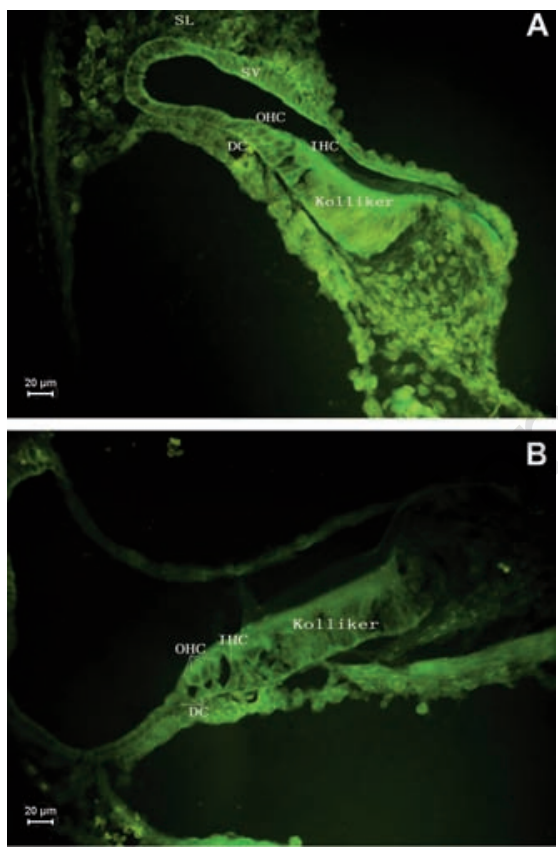

Figure 2. Immunostaining of synaptobrevin 2 in Corti's organ and Kolliker's organ of P1 and P5 group. (A) 200x. There are immature supporting cells and hair cells in Corti's organ and Kolliker's organ. Positive staining of synaptobrevin 2 is not found in Corti's organ and Kolliker's organ in P1 group. (B) 200x. Positive staining of synaptobrevin 2 is not found in Corti's organ and Kolliker's organ in P5 group either.
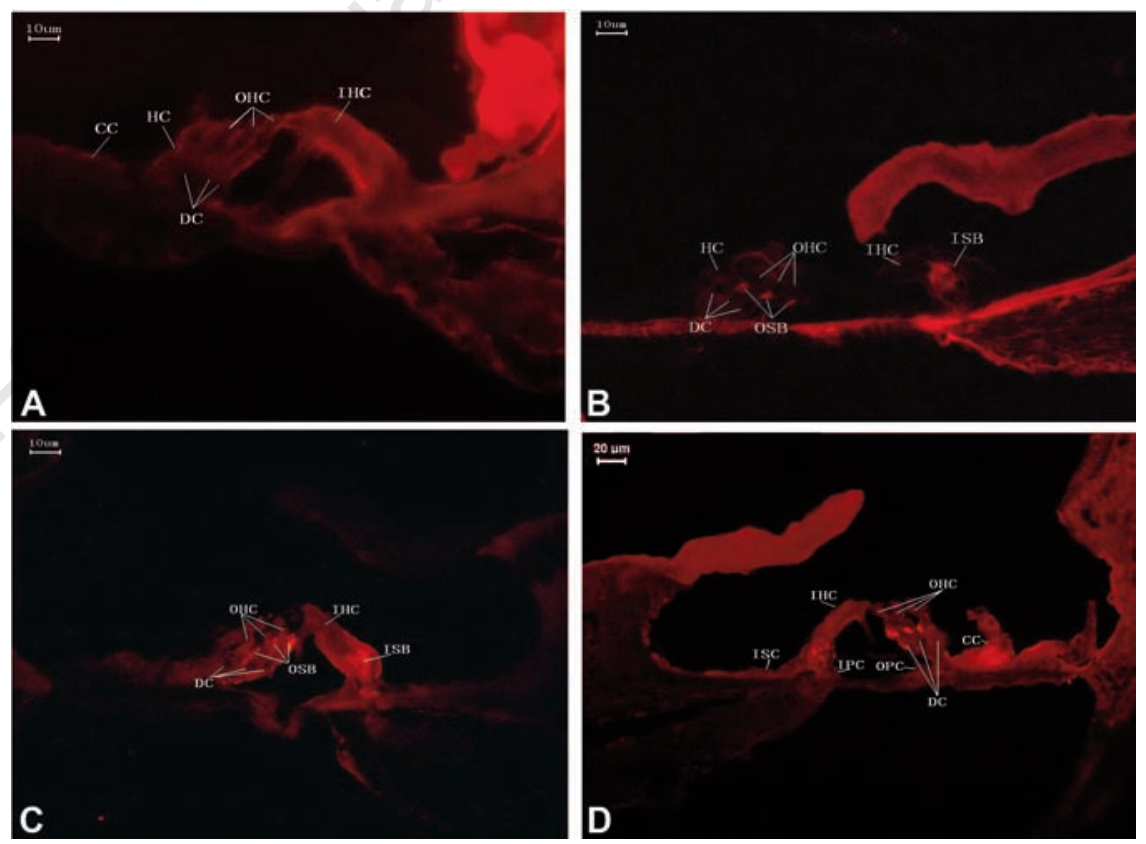

Figure 3. Immunostaining of synaptophysin in Corti's organ of P10, P14 and P28 group. (A) $400 \times$. The Corti's organ of the top turn of P10 has already formed the essential structure, but the expression of synaptophysin did not appear in Corti's organ. (B) 400x. Low immunoreactivity of synaptophysin is seen in the OSB and ISB in the middle turn of cochleae of P10. (C) 400x. The Corti's organ has already matured in P14 group, and the expression of synaptophysin is visible in the OSB, ISB and the medial wall of the Deiters' cell. (D) 200x. Strong punctuate synaptophysin expression is found strong punctate expression in the ISB, and cup-shaped expression is found in the OSB. The association of the tunnel spiral bundle and the ISB, also and the inner medial wall of the Deiter's cell show weak expression. (CC, Claudius' cell; ISB, inner spiral bundle; OSB, outer spiral bundle; IPC, inner pillar cell; OPC, outer pillar cell; ISC, inner sulcus cell; TSB, tunnel spiral bundle). 
P14, Corti's organ had almost reached its mature morphology. Synaptophysin expression was detected in the OSB, the ISB, and the medial wall of the Deiters' cell of the cochlea, which was found to be expressed in a clustered pattern stronger than that at P10 (Figure 3C). At P28, punctate synaptophysin expression was found in the ISB, whereas a cupshaped pattern was present in the OSB of Corti's organ. Expression at this age was the strongest of all the groups (Figure 3D). The association of the channel spiral bundle and
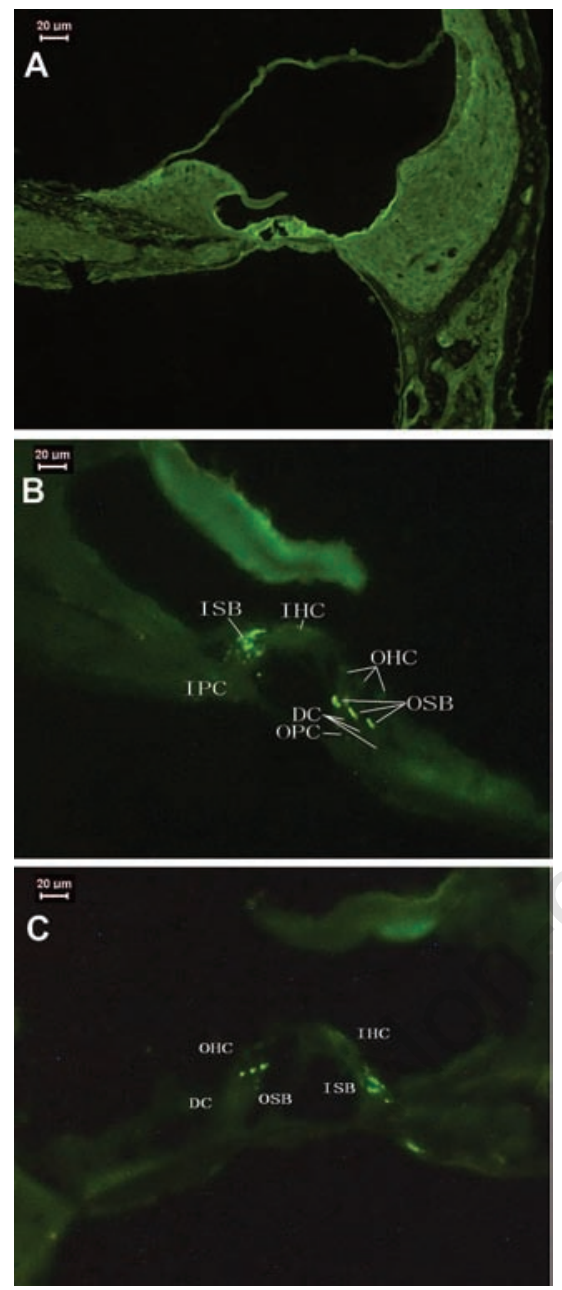

Figure 4. Immunostaining of synaptobrevin 2 in Corti's organ of P10, P14 and P28 group. (A) $200 \times$. The Corti's organ has already formed the essential structure, but the expression of synaptobrevin 2 does not appear in the Corti's organ of P10 group. (B) $400 \times$. The Corti's organ has already matured, and punctuate expression of synaptobrevin 2 is found in the ISB, and cup-shaped expression is found in the OSB. The inner medial wall of the Deiter's cell also showed weak expression. There is punctate expression in the top of the IHCs. (C) $400 \times$. The staining in Corti's organ of P28 is similar to the P14 group. the ISB, and also the inner medial wall of the Deiters' cell, showed weak expression of synaptophsin (Figure 2D).

Synaptobrevin 2 expression in Corti's and Kolliker's organ was not found in the cochleae of P1, P5 or P10 rats (Figures 2 and 4A). The expression sites of synaptobrevin 2 were almost the same as that of synaptophysin in Corti's organ of P10, P14 and P28 rats (Figures 3 and 4 ), and there was punctate expression in the top of the IHCs in Corti's organ of both P14 and P28 rats (Figures 4B and 4C).

\section{Spiral ganglion neurons}

In the P1 and P5 experimental groups, the neurons were arranged closely in a cluster-like pattern, whereas the expression of synaptophysin and synaptobrevin 2 was weaker compared with P10, P14, and P28. By P10 and P14, the phenomenon of clustering was diminished, and the expression of synaptophysin and synaptobrevin 2 was enhanced compared with P1 and P5. By P28, the expression of synaptophysin and synaptobrevin 2 was the
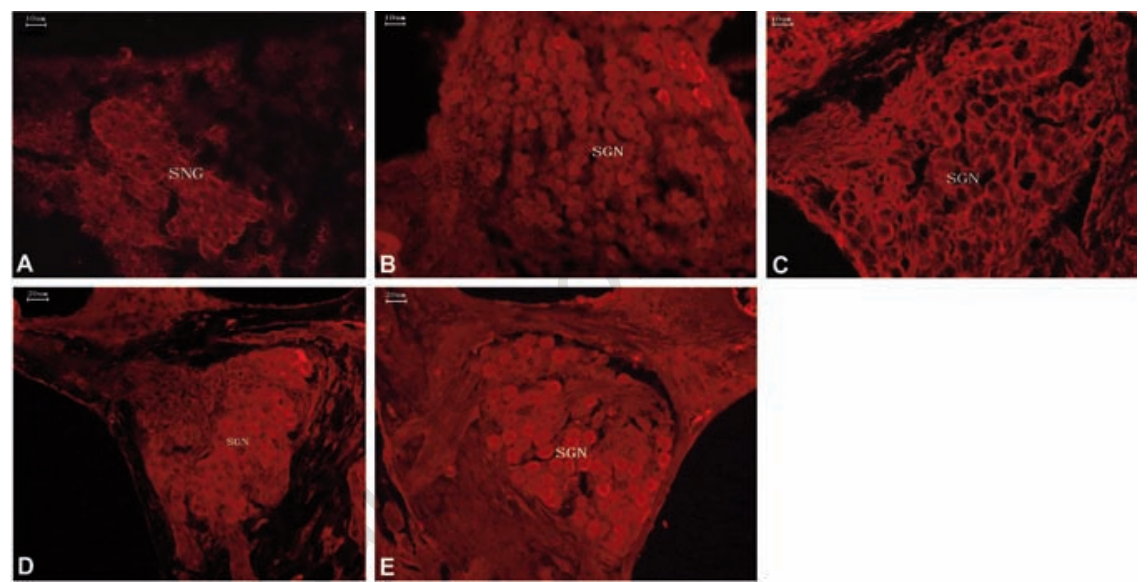

Figure 5. Immunostaining of synaptophysin in SGNs of P1, P5, P10, P14 and P28 group. (A) $400 \times$. SGNs are in clusters, and weak synaptophysin expression is found in the cell body. (B) $400 \times$. The staining in SGNs at P5 is similar to the P1 group (C) 400×. The cells are developmentally mature. The synaptophysin expression in cytoplasm is stronger than P1 and P5 group. (D) 200x. The intercellular space of SGNs becomes large, and expression of synaptophysin is found in the plasma. (E) 200x. The cytoplasm of SGNs shows the strongest synaptobrevin 2 expression at P28.
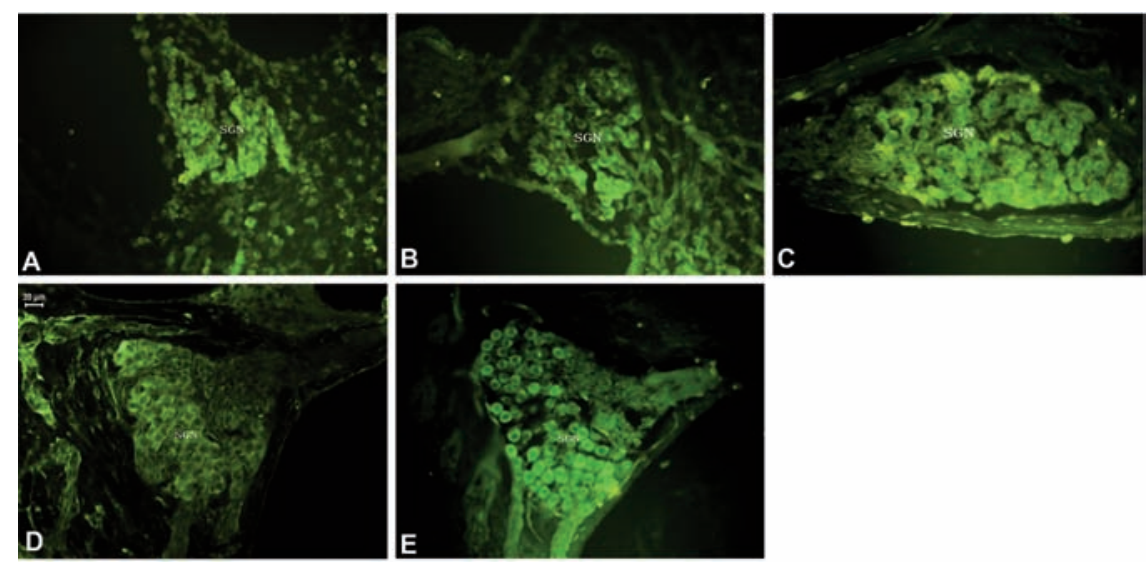

Figure 6. Immunostaining of synaptobrevin 2 in SGNs of P1, P5, P10, P14 and P28 group. (A) $200 \times$. SGNs are in clusters, and weak synaptobrevin 2 expression is found in the cell body. (B) 200x. The staining in SGNs of P5 is similar to the P1 group. (C) $200 \times$. The cells are developmentally mature. The synaptobrevin 2 expression in cytoplasm is stronger than P1 and P5 group. (D) 400x. The intercellular space of SGNs becomes large, and expression of synaptobrevin 2 is found in the plasma. (E) $400 \times$. The cytoplasm of SGNs shows the strongest synaptobrevin 2 expression at P28. 
strongest in all the experimental groups. During the development of the cochleae, all SGNs were stained in different aged cochleae, and the expression of synaptophysin and synaptobrevin 2 in cytoplasm of SGNs was more intense as the cochleae went more mature. The expression of synaptophysin and synaptobrevin 2 was the most intense in P28 group (Figures 5 and 6 ).

\section{Discussion}

\section{Expression of synaptophysin and synaptobrevin 2 in the cochlea and their correlated function}

The development of the mammalian nervous system involves a number of synchronous events, including neurite growth towards appropriate targets, the formation of new synaptic connections, plasticity to reapportion synaptic contacts and axon retraction to remove inappropriate innervation and synapses. In the developing cochlea, these events are likely to play a crucial role in establishing the correct afferent innervation pattern. ${ }^{19-21}$ Before the cochlear afferent innervation reaches its mature configuration, there is an initial mismatch, where both type I and type II SGNs innervate both types of sensory hair cells. During the development of the cochlea, type I SGN innervation is eliminated from the OHCs and type II SGN innervation is eliminated from the IHCs. This reorganization of nerve fibers occurs in rodents after birth and before the onset of hearing..$^{22}$ Meanwhile, the growth and branching of the SGNs is supported by neurotrophic factors secreted by hair cells.

The development of the auditory system is a very complex process, which is subject to detailed mechanisms of regulation and control. In this process, various kinds of structural proteins related to cochlear function are expressed in specific areas of the cochlea. Synaptophysin is a membrane protein specific to synaptic vesicles and correlates directly with the presence of neurotransmitter. Therefore, it also serves as a specific synaptic marker. Synaptobrevin 2 is one of the major components of the SNARE protein complex, and plays a key role in vesicle docking and fusion. Together, synaptophysin and synaptobrevin 2 play an important role in regulating the process of synaptic vesicles exocytosis. Whereas in most vertebrates, the general morphology and structure of the cochleae has been formed prior to birth, and subsequent postnatal periods of cellular differentiation occur before developmental maturity is attained. This is particularly true in rodents, due to the presence of a shorter embryonic period.
Therefore, the cochlea will not reach full maturity until two weeks after birth. ${ }^{18}$ During this process, the differences in cochlear expression of synaptophysin and synaptobrevin 2 have so far not been reported in the literature. The findings of this study therefore allow us to speculate on the functions of synaptophysin and synaptobrevin 2 in the developing cochlea.

\section{Expression of synaptophysin and synaptobrevin 2 in the outer spiral bundle}

It was found that the hair cells and nerve terminals of rat cochleae undergo reorganization during the first week after birth, and the mature mode is not established until the second week. ${ }^{23}$ We found the immunostaining of synaptophsin or synaptobrevin 2 was not observed in P1 or P5 OSB or the apical turn at P10. Expression was consistent in the OSB at P14 and P28 and in the basal or middle turn at P10. The different immunostaining levels at P10 are consistent with the basal-apical gradient. In OSB, the immunostaining of synaptophysin and synaptobrevin 2 was highest at P28, and moderate-tolow from P14 to P10. It was reported that synaptophysin interacts with synaptobrevin 2 only in mature nerve terminals ${ }^{24}$ which was consistent with the absence of synaptophysin and synaptobrevin 2 in Kolliker's organ or Corti's organ at P1, P5 and P10. The differences in the distribution at P10 and P14 in OSB indicated that the mature synaptic connections were established between $\mathrm{OHCs}$ and nerve terminals; this phase was also consistent with the onset of hearing. ${ }^{2}$ The distribution differences between groups showed that the OHCs were controlled by the SGNs, which released neurotransmitters, but the concrete mechanism requires further investigation. Similarly, changes in hair cell function during neurite retraction and synapse pruning may also contribute to variation in synaptic stability. It was reported that glutamate receptors are expressed by type I fibers at this stage of development, ${ }^{25}$ suggesting that these temporary synapses are at least capable of functional neurotransmission with the $\mathrm{OHCs}$.

\section{Expression of synaptophysin and synaptobrevin 2 in the inner spiral bundle}

Most of the SGNs (90-95\%) form synapses on IHCs; more than $95 \%$ of the information transmission in the cochlea relies on IHCs and the synaptic complex, which is regulated by SGNs from the central nervous system (CNS) ${ }^{26}$ We found that the expression of synaptophysin and synaptobrevin 2 was similar within each age group. We were unable to demonstrate expression in the ISB at P1 and P5 and the top turn at P10. They were expressed in the ISB at
P14 and P28 and in the middle and the basal turn at P10. The extent of expression was wider than in the OSB in the same age group. The greater abundance of synaptophsin and synaptobrevin 2 in the ISB is consistent with the fact that IHCs are considered to be more active than OHCs, which confirms that the efferent synapses release neurotransmitters at a synaptic complex with the ISB to produce a marked effect. Glumatic acid and acetylcholine are found to be the main neurotransmitters in the efferent synapse in the synaptic complex below the IHCs ${ }^{27}$ and they play an important role in a variety of physiological functions of the hair cells, such as mechanical-electrical conversion.

\section{Expression of synaptophysin and synaptobrevin 2 in hair cells}

In contrast with the central nervous system, the terminal of hair cells has a specialized structure, the synaptic ribbon. The synaptic vesicles are attached to the synaptic ribbon, through which hair cells can maintain a high and sustained release of neurotransmitters..$^{28}$ Consistent with previous studies, ${ }^{28.30}$ we found that at certain ages hair cells lack synaptophysin. This is also a major difference between cochlear hair cell synapses and CNS synaps$\mathrm{es}^{30}$ and suggests that an unconventional mechanism may be involved in the neurotransmitter release at ribbon synapses.

There was punctate expression in the top of the IHCs in Corti's organ in both P14 and P28 rats, which was different from the synaptophysin expression in hair cells. This may suggest an additional role for synaptobrevin 2 in hair cells, or may simply reflect a targeting mechanism in hair cells that does not involve a direct route to the synaptic area. Synaptophsin/synaptobrevin 2 interaction may have a regulatory role in SNARE complex formation and affect vesicle exocytosis. The absence of synaptophysin, but not synaptobrevin 2, in IHCs raises the possibility of the existence of an unknown synaptophysin isoform, which interacts with synaptobrevin 2 .

\section{Expression of synaptophysin and synaptobrevin 2 in the medial wall of the Deiters' cells}

The clustered expression of synaptophysin and synaptobrevin 2 occurred in the medial wall of Deiters' cells, and the expression, which was gradually weakened from inside to out, was weakest in the medial wall of the lateral Deiter's cells. The expression site of synaptophysin and synaptobrevin 2 at P28, which could be detected only in the medial wall of the medial Deiter's cells, was less intense than that at P14. No immunoreactivity was detected around the supporting cells in other 
age groups. The connection of nerve endings was labeled by synaptophysin and synaptobrevin 2. Deiter's cells mediate signal transduction between nerve endings and cells, indicating that the neural activity of Deiter's cells may be involved in the regulation of auditory function. The changes in the distribution of the expression of synaptophysin and synaptobrevin 2 in different aged groups may indicate that axon retraction and synapse elimination ${ }^{25}$ are required during early postnatal development of cochlea to form correct connections between nerve endings and target cells, and these changes also play an important role in establishing correct encoding of the auditory signals.

\section{Expression of synaptophysin and synaptobrevin 2 in supporting cells}

It has been reported that supporting cells were associated closely with the information transferred between gap junctions that expressed in supporting cells..$^{31,32}$ In this study, no immunoreactivity of synaptophysin and synaptobrevin 2 was detected in supporting cells in different age groups, such as Deiter's cells, Hensen's cells, Claudius cells, and pillar cells. Consistent with previous reports, ${ }^{31,32}$ we speculate that supporting cells in the cochlea might communicate rapidly with each other by gap junctions, rather than by nerve fibers, the same as in cardiac and smooth muscles, which act as a syncytium. Consistent with previous studies ${ }^{33}$ nerve endings were found in the lateral wall of Deiter's cells and Hensen's cells in this study. We discovered that vesicles are packaged by membrane proteins such as synaptophysin and synaptobrevin 2, suggesting that supporting cells are also innervated by nerve fibers. Chemical synapses, which form part of the reflexive circuit loops between supporting cells and OHCs, are formed between nerve endings and Deiter's cells. These loops are important in communication between OHCs and Deiter's cells.

\section{Expression of synaptophysin and synaptobrevin 2 in SGNs}

In rats, apoptosis of SGNs occurs in the embryonic period and in the early postnatal period. This is the same time in which nerve fibers are establishing connections with target cells in the cochlea, such as hair cells. ${ }^{23}$ In our study, all SGNs were immunopositive for both synaptophysin and synaptobrevin 2 in different aged cochleae, and the expression in the cytoplasm of SGNs grew stronger gradually with increasing rat age. The above findings suggest that the apoptosis of SGNs is related to remodeling of axons and synapses affected by OHCs, which are the target cells of type I neurons. This course would lead to the apoptosis of I type neurons in SGNs. Nerve fibers were reported to induce the differentiation and maturation of sensory hair cells and supporting cells. ${ }^{34}$ We found that synaptophysin and synaptobrevin 2 are expressed in SGNs earlier than in hair cells and supporting cells, suggesting that nerve fibers might induce the differentiation and maturation of sensory hair cells and supporting cells. And this induction is probably achieved by releasing certain neurotransmitters such as ATP, which might exist in the vesicles coated by synaptophysin.

\section{The interaction of synaptophysin and synaptobrevin 2}

The course of most vesicles fused with cell membrane is catalyzed by non-specific vesicles. While the docking and fusing course of vesicles, which is a process of targeted vesicle transport, is determined by specific membrane proteins. An increase in intracellular $\mathrm{Ca}^{2+}$ would make the pre-synaptic $\mathrm{Ca}^{2+}$ binding protein transfer information to synaptobrevin $2,^{35}$ which is an important protein in mediating membrane fusion. Synaptobrevin 2 then forms a tight complex with two other plasma membrane proteins, better known as the SNARE complex, which is a prerequisite for membrane fusion. ${ }^{36}$ The SNARE complex would be necessary for the presynaptic membrane and the synaptic vesicle membrane to come into proximity and fuse and further to release neurotransmitter into the synaptic cleft through the fusion pore. ${ }^{37}$ For synaptobrevin 2, the only non-SNARE binding partner protein discovered so far is synaptophysin. The combination of synaptophysin and synaptobrevin 2 can accommodate vesicular exocytosis activity. ${ }^{38}$ In this study, the expression of synaptophysin and synaptobrevin 2 were seen at P14 and P28, and in the middle or the basal turn of Corti's organ at P10. They were expressed in the ISB, OSB, the medial wall of Deiter's cells and the SGNs. Their co-expression suggests that the synaptophysin-synaptobrevin 2 complex might be present in the cochleae of rats.

Synaptophysin is a major protein of the synaptic vesicle membrane, and its binding to synaptobrevin 2 is specific and exclusive. ${ }^{39}$ Synaptobrevin 2 is bound with synaptophysin and cannot enter the SNARE complex. Likewise, synaptobrevin 2 is a part of the SNARE complex and cannot interact with synaptophysin either. These findings lead to the proposal that synaptophysin serves as a control protein that regulates the availability of synaptobrevin 2 for engaging with its SNARE partners before membrane fusion. ${ }^{24}$ The relative instability of the synaptophysin/ synaptobrevin 2 complex may ensure that its formation is readily reversible. The main role of the complex formation is to provide a rapidly avail- able pool of vesicular synaptobrevin 2 for exocytotic membrane fusion. The synaptophysin/ synaptobrevin 2 complex will dissociate in a process driven by ATP, and provide a high volume of synaptobrevin 2 in a very short period of time for the biological activity of neural synapses, and further to form a SNARE protein complex with other membrane proteins. ${ }^{40}$ Then, a fusion pore is formed in the cell membrane at the target sites to prepare for the quantum release of vesicles. ${ }^{41}$ Synaptophysin may fine-tune the synaptic responses by regulating the availability of synaptobrevin 2 to the SNARE complex in a positive manner and by regulating the function of hair cells and supporting cells in cochleae.

\section{References}

1. Lee JH, Heo JH, Kim CH, Chang SO, Kim $\mathrm{CS}, \mathrm{Oh} \mathrm{SH}$. Changes in $\mathrm{P} 2 \mathrm{Y} 4$ receptor expression in rat cochlear outer sulcus cells during development. Hear Res 2007;228:201-11.

2. Auestad N, Stockard-Sullivan J, Innis SM, Korsak R, Edmond J. Auditory brainstem evoked response in juvenile rats fed rat milk formulas with high docosahexaenoic acid. Nutr Neurosci 2003;6:335-41.

3. Powers BE, Widholm JJ, Lasky RE, Schantz SL. Auditory deficits in rats exposed to an environmental PCB mixture during development. Toxicol Sci 2006;89:415-22.

4. Brandt N, Kuhn S, Munkner S, Braig C, Winter H, Blin N, et al. Thyroid hormone deficiency affects postnatal spiking activity and expression of $\mathrm{Ca} 2+$ and $\mathrm{K}+$ channels in rodent inner hair cells. J Neurosci 2007;27:3174-86.

5. Asano-Miyoshi M, Hamamichi R, Emori Y. Synaptophysin as a probable component of neurotransmission occurring in taste receptor cells. J Mol Histol 2009;40:59-70.

6. Yoshida T, Uchida S, Mishina M. Regulation of synaptic vesicle accumulation and axon terminal remodeling during synapse formation by distinct $\mathrm{Ca}$ signaling. J Neurochem 2009;111:160-70.

7. Glueckert R, Bitsche M, Miller JM, Zhu Y, Prieskorn DM, Altschuler RA, et al. Deafferentation-associated changes in afferent and efferent processes in the guinea pig cochlea and afferent regeneration with chronic intrascalar brain-derived neurotrophic factor and acidic fibroblast growth factor. J Comp Neurol 2008;507: 1602-21.

8. Barszczewski M, Chua JJ, Stein A, Winter U, Heintzmann R, Zilly FE, et al. A novel site of action for alpha-SNAP in the SNARE conformational cycle controlling mem- 
brane fusion. Mol Biol Cell 2008;19:776-84.

9. Elfving B, Bonefeld BE, Rosenberg R, Wegener G. Differential expression of synaptic vesicle proteins after repeated electroconvulsive seizures in rat frontal cortex and hippocampus. Synapse 2008;62:662-70.

10. Baldwin MR, Barbieri JT. Association of botulinum neurotoxins with synaptic vesicle protein complexes. Toxicon 2009;54: 570-4.

11. Raptis A, Torrejon-Escribano B, Gomez de Aranda I, Blasi J. Distribution of synaptobrevin/VAMP 1 and 2 in rat brain. J Chem Neuroanat 2005;30:201-11.

12. Nemzou NR, Bulankina AV, Khimich D, Giese A, Moser T. Synaptic organization in cochlear inner hair cells deficient for the CaV1.3 (alpha1D) subunit of L-type Ca2+ channels. Neuroscience 2006;141:1849-60.

13. Khalifa SA, Friberg U, Illing RB, RaskAndersen H. Synaptophysin immunohistochemistry in the human cochlea. Hear Res 2003;185:35-42.

14. Matsumoto M, Nakagawa T, Kojima K, Sakamoto T, Fujiyama F, Ito J. Potential of embryonic stem cell-derived neurons for synapse formation with auditory hair cells. J Neurosci Res 2008;86:3075-85.

15. Bartolome MV, Zuluaga P, Carricondo F, Gil-Loyzaga P. Immunocytochemical detection of synaptophysin in C57BL/6 mice cochlea during aging process. Brain Res Rev 2009;60:341-8.

16. Sendin G, Bulankina AV, Riedel D, Moser T. Maturation of ribbon synapses in hair cells is driven by thyroid hormone. $\mathrm{J}$ Neurosci 2007;27:3163-73.

17. Layton MG, Robertson D, Everett AW, Mulders WH, Yates GK. Cellular localization of voltage-gated calcium channels and synaptic vesicle-associated proteins in the guinea pig cochlea. J Mol Neurosci 2005; 27:225-44.

18. Montcouquiol M, Kelley MW. Planar and vertical signals control cellular differentiation and patterning in the mammalian cochlea. J Neurosci 2003;23:9469-78.

19. Montgomery JM, Madison DV. Discrete synaptic states define a major mechanism of synapse plasticity. Trends Neurosci 2004;27:744-50.

20. Sugihara I. Organization and remodeling of the olivocerebellar climbing fiber projection. Cerebellum 2006;5:15-22.

21. Malenka RC. The long-term potential of LTP. Nat Rev Neurosci 2003;4:923-6.

22. Housley GD, Bringmann A, Reichenbach A. Purinergic signaling in special senses. Trends Neurosci 2009;32:128-41.

23. Nikolic P, Jarlebark LE, Billett TE, Thorne PR. Apoptosis in the developing rat cochlea and its related structures. Brain Res Dev Brain Res 2000;119:75-83.

24. Reisinger C, Yelamanchili SV, Hinz B, Mitter D, Becher A, Bigalke H, et al. The synaptophysin/synaptobrevin complex dissociates independently of neuroexocytosis. J Neurochem 2004;90:1-8.

25. Huang LC, Thorne PR, Housley GD, Montgomery JM. Spatiotemporal definition of neurite outgrowth, refinement and retraction in the developing mouse cochlea. Development 2007;134:2925-33.

26. Puel JL, Ruel J, Guitton M, Wang J, Pujol R. The inner hair cell synaptic complex: physiology, pharmacology and new therapeutic strategies. Audiol Neurootol 2002;7:49-54.

27. Housley GD, Jagger DJ, Greenwood D, Raybould NP, Salih SG, Jarlebark LE, et al. Purinergic regulation of sound transduction and auditory neurotransmission. Audiol Neurootol 2002;7:55-61.

28. Safieddine S, Wenthold RJ. SNARE complex at the ribbon synapses of cochlear hair cells: analysis of synaptic vesicle- and synaptic membrane-associated proteins. Eur J Neurosci 1999;11:803-12.

29. Jongkamonwiwat N, Phansuwan-Pujito P, Casalotti S0, Forge A, Dodson $\mathrm{H}$, Govitrapong P. The existence of opioid receptors in the cochlea of guinea pigs. Eur J Neurosci 2006;23:2701-11.

30. Li M, Cui Z, Niu Y, Liu B, Fan W, Yu D, et al. Synaptogenesis in the developing mouse visual cortex. Brain Res Bull 2010;81:10713.

31. Zhao HB, Yu N, Fleming CR. Gap junctional hemichannel-mediated ATP release and hearing controls in the inner ear. Proc Natl
Acad Sci USA 2005;102:18724-9.

32. Liu W, Bostrom M, Kinnefors A, RaskAndersen H. Unique expression of connexins in the human cochlea. Hear Res 2009;250:55-62.

33. Furness DN, Hulme JA, Lawton DM, Hackney CM. Distribution of the glutamate/aspartate transporter GLAST in relation to the afferent synapses of outer hair cells in the guinea pig cochlea. J Assoc Res Otolaryngol 2002;3:234-47.

34. Kelley MW. Regulation of cell fate in the sensory epithelia of the inner ear. Nat Rev Neurosci 2006;7:837-49.

35. Bretou M, Anne C, Darchen F. A fast mode of membrane fusion dependent on tight SNARE zippering. J Neurosci 2008;28: 8470-6.

36. Song H, Nie L, Rodriguez-Contreras A, Sheng ZH, Yamoah EN. Functional interaction of auxiliary subunits and synaptic proteins with $\mathrm{Ca}(\mathrm{v}) 1.3$ may impart hair cell $\mathrm{Ca} 2+$ current properties. J Neurophysiol 2003;89:1143-9.

37. Sorensen JB. Conflicting views on the membrane fusion machinery and the fusion pore. Annu Rev Cell Dev Biol 2009; 25513-37.

38. Becher A, Drenckhahn A, Pahner I, Margittai M, Jahn R, Ahnert-Hilger G. The synaptophysin-synaptobrevin complex: a hallmark of synaptic vesicle maturation. J Neurosci 1999;19:1922-31.

39. Bonanomi D, Rusconi L, Colombo CA, Benfenati F, Valtorta F. Synaptophysin I selectively specifies the exocytic pathway of synaptobrevin 2/VAMP2. Biochem J 2007;404:525-34.

40. Mima J, Wickner W. Phosphoinositides and SNARE chaperones synergistically assemble and remodel SNARE complexes for membrane fusion. Proc Natl Acad Sci USA 2009;106:16191-6.

41. Ito H, Atsuzawa K, Morishita R, Usuda N, Sudo K, Iwamoto I, et al. Sept8 controls the binding of vesicle-associated membrane protein 2 to synaptophysin. J Neurochem 2009;108:867-80. 
